of this important class of substances. His, again, is a comprehensive review, and he is able to rationalize the experimental results in terms of the coordination number of the phosphorus atoms and the state of hybridization of the carbon atoms to which they are attached. This review also contains, in the section on cyclopolyphosphines, an illuminating account of the geometrical constraints that are inherent in non-planar polygons.

These four articles form a valuable addition to the literature on structural chemistry. They are eminently readable, and the standard of production is high. The volume also contains a useful cumulative subject index. One is left with the strong impression (and this is reinforced by the fact that two of the authors have considered it necessary to give addenda listing work carried out after the articles were submitted) that the various areas are expanding rapidly. This in turn suggests that there should be no shortage of articles for future volumes and I, for one, shall look forward to them.

Department of Physical Chemistry

P. J. Wheatley

Lensfield Road

Cambridge CB2 $1 E P$

England

Models in structural inorganic chemistry. By A.F. Wells. Pp.xi +186 . London: Oxford University Press, 1970. Price (hard cover) $£ 2 \cdot 75$, (paper cover) $£ 1 \cdot 40$.

This is an unusual book. It is not only a small treatise in its own right, but it can be regarded as a practical manual to the author's large Structural Inorganic Chemistry. It is meant to be used with a special model kit, which consists of (1) a set of plastic tubes and connectors for the construction of polyhedra,

(2) drilled balls and spokes, the balls each having the usual 26 holes,

(3) 'space-filling' balls, with some joined together in groups and some to fit into the intertices of these,

(4) polyhedra, either single tetrahedra and octahedra, or units with shared edges. Special connectors for these are also supplied.

The chapter on Polyhedra and other Finite Systems contains a number of problems, for example that numbered P5 is as follows: 'With 8 rods of equal length construct a halfoctahedron (square pyramid). An atom placed at the centre of the base is equidistant from the five vertices. Now insert a ninth rod, of the same length as the other eight, as one diagonal of the base. The figure becomes a a trigonal bipyramid.' Other problems include a detailed study of the conformation of 6 and 8 membered rings which would be of value to students of stereochemistry. Another interesting point brought out in this chapter is the distinction between cis-trans isomerism in a molecule and enantiomorphism.

The next chapter provides a series of problems on Networks (N1 to N18). Appendices 2 and 3 of the book outline some of the author's previously published theories. The problems cover many important structural types such as those of the diamond, wurtzite, borates, etc. Such network theory would seem to be of great value as a possible means of classification of structures, perhaps the only logical geometrical method. However, even this method breaks down owing to the fact that atoms have many different strengths of bonds whilst network theory is essentially an 'all or nothing' procedure.

Chapter 3 deals with the packing of spheres, and contains problems S1 to S18. The questions investigated include the simple close-packed structures of equal atoms, and their interstitial structures. An interesting packing which has a fivefold symmetry axis and a density only slightly less than the close-packed one is described. Is it possible that this is related to the fivefold symmetry common in dicotyledonous plants? The whole subject is discussed in an interesting and stimulating way.

Subsequent chapters deal with structures built from tetrahedra and from octahedra, thus dealing among other things with silicates, and molybdates and tungstates. It will surprise most readers to discover what complex structures can be dealt with and understood with the simple units available. It should however be pointed out that there are important features in some structures which are ignored in models made from regular polyhedra, e.g. the distortion of the octahedra surrounding the aluminum atoms in corundum, due to metal-metal bonding. A few miscelalneous structures are dealt with in Chapter 6 (Problems M1 to M6). The remaining chapters 7 to 12 give the Answers to all the problems in the earlier chapters together with much additional information. The book concludes with some appendices which outline some of the author's previous contributions to the theory of polyhedra and of networks.

Although the book is quite short it is a most thoughtful and thought-provoking one, and is a considerable contribution to the understanding of structural inorganic chemistry. It is beautifully produced and almost every opening has its clear and original diagrams. Chemists, mineralogists and even physicists and metallurgists should keep it on their shelves, where it would be of permanent value - the structures described are not likely to change very much, even less the underlying geometry! The model kit which goes with the book is rather expensive. Most users may contrive with their own cork and toothpick methods of the past!
Chemistry Department
University of Edinburgh
Edinburgh
Scotland

\section{A. BeEvers}

